\title{
Measuring temperature using MRI: a powerful and versatile technique
}

\author{
Robert Turner $\cdot$ Markus Streicher
}

Published online: 6 January 2012

(C) ESMRMB 2011

\begin{abstract}
The Larmor frequency of water protons has reliably linear temperature dependence. Since this frequency shift is easily measurable using relatively simple MRI techniques, a remarkable opportunity arises for uniquely non-invasive and accurate temperature evaluation, deep within any water-containing object. Major applications are appearing in the field of image-guided surgery. The cutting-edge papers collected in this Special Issue demonstrate both the versatility and the power of MRI thermometry.
\end{abstract}

Keywords MR thermometry · Proton resonance frequency shift · Magnetic susceptibility · MR-guided intervention

\section{Introduction}

It has often been remarked that water is a highly unusual liquid. Apart from being very abundant and liquid at average Earth planetary temperature, it has an exceptionally high specific heat and latent heats of transition, its molecules are polar, it has strong optical absorption at infrared and ultraviolet frequencies, and it exhibits hydrogen bonding. Almost all these unusual properties, without which life would not be possible, are related to the fact that the $\mathrm{H}_{2} \mathrm{O}$ molecule is not straight, with a hydrogen atom on either side of the oxygen atom, but in its ground state of energy, it is bent, with an angle of about $105^{\circ}$ between the two bonds.

R. Turner $(\bowtie) \cdot$ M. Streicher

Max-Planck Institute for Human Cognitive and Brain Sciences, Stephanstraße 1A, 04103 Leipzig, Germany

e-mail: turner@cbs.mpg.de
This simple fact also has an important effect on the Larmor resonance frequency of the protons that constitute the hydrogen nuclei. If a magnetic field is externally applied to water, its protons experience a resultant magnetic field, the sum of the external field and the shielding field arising from the response of the molecule's mobile electrons. Each proton's resonant frequency is determined by this net magnetic field through the Larmor equation. Hydrogen bonds between neighboring molecules distort the electronic configuration of the molecules and reduce the electronic screening of the hydrogen proton. The fraction and nature of hydrogen bonds change with temperature, and on average, the water molecules spend less time in the hydrogen-bonded state. As a result, the electronic screening increases, and hence, the resultant magnetic field experienced by the hydrogen proton is decreased, leading to a decrease in Larmor frequency with increasing temperature. This temperature dependence is easily measurable at typical Larmor frequencies of $60 \mathrm{MHz}$ and above. It has been found to be linear over a usefully large temperature range, giving rise to the proton resonance frequency (PRF) method for NMR temperature measurement [1-3].

By contrast, many other protons (typically in compounds with $\mathrm{CH}$ bonds) giving a resonance line in NMR spectra are not involved in hydrogen bonds. Thus, their resonance frequencies have much smaller temperature dependence. If these resonances are sufficiently distinct from the water proton resonant frequency, such compounds can provide a temperature-independent reference to correct for systematic errors in MR thermometry [4].

The main current areas of application of MR thermometry are the monitoring of minimally invasive image-guided surgery and assessment of the safety of radiofrequency transmit coils by measuring temperature change. Both applications benefit remarkably from the 
accuracy and volumetric character of the temperature measurement.

A recent review by Tempany et al. [5], discussing surgery based on focused ultrasound, argues convincingly that the opportunity for simultaneous excellent soft-tissue contrast and non-invasive real-time thermometry makes MR the most reliable and comprehensive modality available to guide therapeutic ultrasound. For this reason, MR thermometry has found clinical applications in recent years (fibroid ablation) and other numerous potential fields of application. Not only is focused ultrasound compatible with MRI, but so too are cryoablation and laser ablation. A widely shared goal is to make MR thermometry accurate enough to be able to apply it practically anywhere in the human body, whether close to an air-tissue interface, in fatty tissue or in fast-moving organs such as the heart.

Besides the PRF-shift method, several parameters accessible to MRI measurement are temperature sensitive, including the apparent diffusion coefficient $\mathrm{D}$, the $\mathrm{T}_{1}$ and $\mathrm{T}_{2}$ relaxation times, the magnetization transfer rate, as well as the effect of temperature-sensitive contrast agents. However, the excellent linearity and temperature dependence of PRF have made PRF-shift-based phase mapping methods the preferred choice for many in vivo applications [2]. One weakness of this method is that even very small changes in local magnetic field strength can be misinterpreted as temperature changes.

\section{Contents}

This Special Issue of MAGMA contains seven papers advancing the technical capabilities of MR thermometry in different ways. Six are related directly to interventional applications, and the seventh deals with an unexpected potential source of measurement error, the small but measurable effect of changes in the magnetic susceptibility of the surrounding air, due to the changes in temperature and composition. A common theme of these papers is the origin of local magnetic field changes and methods to correct for them. They may arise from organ movement $[6,7]$, tissue susceptibility changes due to freezing [8] or fat susceptibility change due to the temperature changes [9]. They may even be caused by susceptibility changes in surrounding air [10].

Wyatt et al. [11] here focus on finding the optimal echo time combinations for fat-water voxels to minimize temperature noise in multi-echo sequences. Hoffmann et al. [12] discuss the topic of residual tumors after thermal ablation, which is of importance for the application of thermal ablation to malignant tumors.

In general, MR thermometry using fast gradient echo sequences or echo-planar imaging, either segmented or single shot, is fast enough to acquire large enough ROIs for interventions. However, for the special application of MR thermometry in the heart, Mei et al. [6] developed an extremely fast 1D spin echo sequence with a temporal resolution of up to $19 \mathrm{~Hz}$.

Using a different strategy, Maier et al. [7] introduce a fast velocity-navigator motion-compensated MR sequence that can be triggered by a velocity in any direction. In their publication, the sequence was triggered by the point of full expiration using a Kalman filter to suppress cardiac spikes. After the trigger event, the whole slice was acquired, giving temperature maps similar to those obtained during a breath-hold acquisition. This enables the treatment of less compliant patients, which may often be the case in situation in which thermal therapies make sense.

More subtle magnetic field changes than those due to motion occur in the case of susceptibility changes of tissue caused by temperature changes. As Sprinkhuizen et al. [13] have shown previously in a study of fatty breast tissue, a temperature change of $30 \mathrm{~K}$ can lead to a PRF-shift temperature error of almost $10 \mathrm{~K}$. To better model and ideally to correct these fat susceptibility changes, they measured [9] the magnetic volume susceptibility change with the temperature of breast fat tissue samples from six donors. They found a linear dependence of the fat susceptibility with temperature, although the temperature coefficient varied widely from 0.0039 to $0.0076 \mathrm{ppm} / \mathrm{K}$, and the absolute susceptibility values also showed a wide range. The authors concluded that this large variation might be due to different compositions of fat tissue, with different amounts of blood and liquid matrix contamination, and possibly even different fatty acid composition.

It may be possible to correct for the errors induced by fat susceptibility changes using a variant of the algorithm of Kickhefel et al. [8], introduced in order to correct for the magnetic perturbation field caused by the frozen tissue in cryoablation. Their algorithm assumes a uniform temperature on the boundary surface between frozen and unfrozen tissue and then estimates the susceptibility difference for the frozen and unfrozen tissue-frozen tissue has a higher magnetic susceptibility. By this means, they were able to correct for potential PRF temperature errors of about $10 \mathrm{~K}$. This improved accuracy might help to protect important tissue structures in proximity to the ablation zone by correctly monitoring the temperature, which cannot be done by other methods.

Another source of error in MR thermometry is the susceptibility change of surrounding air or gas. Streicher et al. [10] showed in measurements and simulations that air susceptibility change with temperature has a measurable effect on uncorrected MR thermometry and an even stronger effect when oxygen concentration is changed. This effect can lead to PRF temperature errors of up to $40 \mathrm{~K}$. 
Both observations are related to the paramagnetism of the oxygen molecule, whose magnetic susceptibility is strongly temperature dependent and dominates all other diamagnetic components of air.

Along these lines, Wyatt et al. [11] found optimal sequence parameters for multi-echo MR thermometry sequences, applying the Cramer-Rao lower bound analysis to make MR thermometry as accurate as possible. They used this analysis on multi-echo sequences on fat-water voxels, which included three distinct fat peaks. The results were then very convincingly verified by experiment and compared with those of the much less efficient MonteCarlo simulation method.

For MR-guided thermal ablation to become common clinical practice, it is important to know the ablation parameters necessary to completely coagulate the tissue. Hoffmann et al. [12] performed a thorough study of laser ablation of lung cancer using an ex vivo lung perfusion and ventilation model. They found that the coagulation size correlated with the maximum temperature which was reached during ablation and also with how long the tissue was heated to temperatures above $44^{\circ} \mathrm{C}$.

Summarizing this Special Issue, it is clear that many MR thermometry techniques, well adapted to a range of clinical contexts, are now 'ready for use', at a stage when clinical trials will be most welcome to establish this unique tool in routine practice.

\section{References}

1. Hindman JC (1966) Proton resonance shift of water in gas and liquid states. J Chem Phys 44(12):4582-4592

2. Rieke V, Pauly KB (2008) MR thermometry. J Magn Reson Imaging 27(2):376-390
3. Quesson B, de Zwart JA, Moonen CTW (2000) Magnetic resonance temperature imaging for guidance of thermotherapy. J Magn Reson Imaging 12(4):525-533

4. Kuroda K, Oshio K, Chung AH, Hynynen K, Jolesz FA (1997) Temperature mapping using the water proton chemical shift: a chemical shift selective phase mapping method. Magn Reson Med 38(5):845-851

5. Tempany CMC, McDannold NJ, Hynynen K, Jolesz FA (2011) Focused ultrasound surgery in oncology: overview and principles. Radiology 259(1):39-56

6. Mei C-S, Mulkern R, Oshio K, Chen N-K, Madore B, Panych L, Hynynen K, McDannold N (2011) Ultrafast 1D MR thermometry using phase or frequency mapping. Magn Reson Mater Phy 25(1):5-14

7. Maier F, Krafft A, Yung J, Stafford R, Elliott A, Dillmann R, Semmler W, Bock M (2011) Velocity navigator for motion compensated thermometry. Magn Reson Mater Phy 25(1):15-22

8. Kickhefel A, Weiss C, Roland J, Gross P, Schick F, Salomir R (2011) Correction of susceptibility-induced GRE phase shift for accurate PRFS thermometry proximal to cryoablation iceball. Magn Reson Mater Phy 25(1):23-31

9. Sprinkhuizen S, Bakker C, Ippel J, Boelens R, Viergever M, Bartels (2011) Temperature dependence of the magnetic volume susceptibility of human breast fat tissue: an NMR study. Magn Reson Mater Phy 25(1):33-39

10. Streicher M, Schäfer A, Reimer E, Dhital B, Trampel R, Ivanov D, Turner R (2011) Effects of air susceptibility on proton resonance frequency MR thermometry. Magn Reson Mater Phy 25(1):41-47

11. Wyatt C, Soher B, Arunachalam K, MacFall J (2011) Comprehensive analysis of the Cramer-Rao bounds for magnetic resonance temperature change measurement in fat-water voxels using multi-echo imaging. Magn Reson Mater Phy 25(1):49-61

12. Hoffmann C, Rosenberg C, Linder A, Hosten N (2011) Residual tumor after laser ablation of human non-small-cell lung cancer demonstrated by ex vivo staining: correlation with invasive temperature measurements. Magn Reson Mater Phy 25(1):63-74

13. Sprinkhuizen SM, Konings MK, van der Bom MJ, Viergever MA, Bakker CJG, Bartels LW (2010) Temperature-induced tissue susceptibility changes lead to significant temperature errors in PRFS-Based MR thermometry during thermal interventions. Magn Reson Med 64(5):1360-1372 